\section{The Therapeutic Potential of Cannabinoids and Phonopho- resis in Pain Management: A Scoping Review}

\section{Carlneshia Hunnicutt ${ }^{1 *}$, Emily Grullón ${ }^{1}$, MeLisa Morrison ${ }^{1}$, Inez Lomeli' ${ }^{1}$, Kurt Hubbard ${ }^{2}$}

${ }^{1}$ Entry-Level Doctor of Occupational Therapy Program, Nova Southeastern University, Florida, USA

${ }^{2}$ Executive Director/ Academic Dean of Online Learning, Remington College, Florida, USA

\begin{abstract}
Background: Cannabis has been an area of interest within the healthcare field due the plant's medicinal properties. Although there is controversy within the United States regarding potential psychoactive effects of cannabinoids, there is no denying the fast-acting analgesic properties. A common method used for pain management is phonophoresis, a physical agent modality utilized by occupational therapy practitioners and other healthcare professionals within a rehabilitation setting. Cannabinoids may be broken down into a form that can be used by phonophoresis, to potentially facilitate pain management, thus positively impacting therapeutic effects.
\end{abstract}

Objective: A scoping review that investigates the effectiveness of phonophoresis and cannabinoids in pain management. Additionally, the introduction of a potential therapeutic technique that combines both phonophoresis and cannabinoid pharmacological agents from a biomechanical frame of reference to address pain and improve the standard of care treatment is paramount.

Conclusion: Cannabis has been used for medicinal purposes for over 5,000 years and phonophoresis has been utilized to deliver pharmacological agents. Therefore, transdermal delivery of pharmacological cannabinoids through phonophoresis may be possible through nano-emulsifications of delta-9 Tetrahydrocannabinol (THC)

*Corresponding author: Carlneshia Hunnicutt, Entry-Level Doctor of Occupational Therapy Program, Nova Southeastern University,Florida,USA, Tel: +1 305 240 7555; E-mail: HunnicuttC@live.com

Citation: Hunnicutt C, Grullón E, Morrison M, Lomeli I, Hubbard K (2019) The Therapeutic Potential of Cannabinoids and Phonophoresis in Pain Management: A Scoping Review. J Phys Med Rehabil Disabil 5: 036.

Received: August 015, 2019; Accepted: October 03, 2019; Published: October 10,2019

Copyright: ( 2019 Hunnicutt C, et al. This is an open-access article distributed under the terms of the Creative Commons Attribution License, which permits unrestricted use, distribution, and reproduction in any medium, provided the original author and source are credited. and Cannabidiol (CBD). The therapeutic use of cannabinoids is a relatively new area and further research is recommended. Additionally, research on phonophoresis has demonstrated inconclusive results associated with pain management.

Keywords: Cannabidiol; Cannabinoids; Cannabinoids in pain management; Pain management; Phonophoresis; Phonophoresis in pain management

\section{Introduction}

Cannabinoids derived from the Cannabis sativa and Cannabis indica plants consists of medicinal and therapeutic properties which have been utilized for over 5 millennia in various cultures to treat malaria, constipation, and rheumatic pains. Additionally, cannabis was used as a muscle-relaxant, anticonvulsant, antiemetic, and an analgesic [1,2]. However, despite the available evidence regarding the medicinal and therapeutic properties of cannabis, it was removed from the US Pharmacopoeia in 1941 due to the drug's perceived associations with crimes [1]. In fact, it is only over the past few years that legalization efforts within the United States have expanded. Currently, medicinal marijuana is legalized in 33 US states, the District of Columbia, Guam, and Puerto Rico [3].

The most commonly used cannabinoids are delta-9-tetrahydrocannabinol (THC) and Cannabidiol (CBD). THC consists of psychoactive properties and is the most abundant cannabinoid found within the cannabis plant, whereas CBD is minimally psychoactive and the second most abundant [1].Both THC and CBD have specific actions within the Endocannabinoid System (ECS) at the Cannabinoid type 1 (CB1) and cannabinoid type 2 (CB2) receptors, which were discovered 30 years ago $[2,4,5]$. The ECS is a complex system of receptors, ligands, and enzymes, which aid in the regulation of fundamental processes at the level of the central andperipheral nervous systems [6]. An abundance of $\mathrm{CB} 1$ receptors can be found in the brain and peripheral tissues; whereas CB2 receptors are expressed in the immune and hematopoietic systems $[1,2,7,8]$. Cannabinoid type 1 and cannabinoid type 2 receptors are involved in the immune and nervous system process, such as inflammation, neural development and pain [2]. Cannabinoid and opioid receptors are believed to have an influence on the spinal level modulation of peripheral pain inputs [5].

Phonophoresis is a type of ultrasound application used to deliver pharmacological agents transdermally to superficial and deep tissue [9]. Phonophoresis was initially used in 1954 to treat polyarthritis on the hand through transdermal delivery of hydrocortisone ointment into areas of inflammation and has been used in clinical medicine for over 60 years $[10,11]$. Research supports the effectiveness of phonophoresis in treating multiple conditions such as epicondylitis, tendinitis, tenosynovitis, bursitis, and osteoarthritis [12]. Phonophoresis has been demonstrated to be more beneficial over oral administration of drugs, due to less side effects, more accurate dosage, minimal adverse interactions and more cost effectiveness [10].

The transdermal delivery of cannabinoids is intended to benefit patients with soft tissue related injuries by targeting peripheral sites. 
Ultimately, the goal is to increase action time of the analgesic $(\mathrm{CBD} /$ THC) to optimize function and improve the standard of care treatment, specifically to facilitate therapeutic gains. The hope is that therapists will take more of an active role in pain management by providing a non-invasive treatment utilizing a Physical Agent 88 Modality (PAM). The reduction of peripheral pain will aid in the promotion of functional activities that are important to patients. The transdermal delivery of $\mathrm{CBD} / \mathrm{THC}$ via phonophoresis is hypothesized to aid in the reduction of peripheral pain, thus promoting occupational engagement and improvement in quality of life.

Therefore, a logical and proposed extension to assist in pain management is the transdermal delivery of $\mathrm{CBD} / \mathrm{THC}$ using phonophoresis to promote greater efficacy for patients in treatment as well as potentially reducing unwanted side-effects of traditional pain medication use.

\section{Methods}

Due to the absence of research on the transdermal delivery of cannabinoids through phonophoresis, a scoping review was conducted utilizing the five stages developed by Arskey and O'Malley [13]. A review of the evidenceregarding the benefits of cannabinoids and phonophoresis for pain management published between 2007-2017 was conducted in order to address how the proposed technique may be implemented. Therefore, the scoping review aims to answer the following questions:

1. Is there a potential for cannabinoids to be transdermally delivered through phonophoresis?

2. What is the efficacy of cannabinoids on pain management?

3. What is the efficacy of phonophoresis on pain management?

4. What is occupational therapy's role with this proposed therapeutic technique?

To meet the inclusion criteria, the literature needed to address pain, the benefits of THC and CBD, and the benefits of phonophoresis. Articles were excluded if they were published prior to 2007 , non-human preclinical phonophoresis studies, non-human preclinical THC studies, and focused on the psychoactive properties of cannabinoids. (Table 1) displays the electronic databases and key terms used. The search yielded a total of 1,026 results. A total of 30 articles met the inclusion and exclusion criteria and were used in the scoping review.

\begin{tabular}{|c|c|}
\hline Databases & Key Terms \\
\hline & Cannabidiol \\
\hline \multirow{2}{*}{ CINAHL } & Pain \\
\cline { 2 - 2 } & Cannabis \\
\hline MedLine & Sonophoresis \\
\hline Google Scholar & Phonophoresis \\
\hline Other Sources & CBD marijuana \\
\hline & Topical CBD \\
\hline & THC \\
\hline & Delta-9-tetrahydrocannabino \\
\hline
\end{tabular}

Table 1: Summary of the Search Strate.

\section{Results}

\section{Medicinal use of Delta-9 cannabinol and cannabidiol}

Delta-9-tetrahydrocannabinol (THC) has been shown to be effective in the reduction of spasticity in patients with Multiple Sclerosis (MS) [14]. In Germany and the United Kingdom (U.K.), THC is included in the treatment for symptom improvement in adult patients with moderate to severe spasticity resulting from MS [14]. The Summary of Product Characteristics (SPC) in the U.K. recommends an oro-mucosal THC/CBD spray to be self- administered for a fourweek treatment of adults with moderate to severe spasticity[14]. A $\geq 20 \%$ of reduction in spasticity during the four-week trial was utilized as a reliable predictor of continued response to THC/CBD[14]. Additionally, in Germany this same treatment of THC/CBD oro-mucosal spray was considered to be more cost effective when compared to anti-spasticity treatment. Therefore, THC/CBD oro-mucosal spray may be more accessible to individuals with spasticity, regardless of socioeconomic status[14].

Drossel, Forchheimer, and Meade [15] conducted a survey addressing cannabis for therapeutic purposes (CTP) for individuals with spinal cord injuries (SCI). It was found that CTP was used regularly and commonly used to control bowel/bladder management, spasticity, pain, and anxiety. Participants stated cannabinoids was used primarily for pain relief. The data suggested that CTP did not alleviate prescriptions for other medications that treat pain symptoms related to SCI. Instead, the CTP worked as a supplemental treatment [15].

Similarly, Rajesh et al. [16], addressed the benefits of CBD in treating diabetic complications and atherosclerosis. Atherosclerosis has the potential to cause strokes, myocardial infarction, and peripheral artery disease. The early stages of atherosclerosis involve increased adhesion of monocytes to endothelium, with eventual progressive migration into the subendothelial space. High glucose (HG) concentration increases the surface expression of these adhesion molecules while CBD decreases or weakens the effects of HG [16].

Additionally, CBD oil is beneficial in the treatment of autoimmune diseases, due to its anti-146 inflammatory properties, [17]. Hammell et al. [18], found significant evidence on CBD's ability to reduce joint swelling, thus improving one's functional condition.Recently, researchers examined the effects of CBD in the treatment of anxiety and found that CBD can be beneficial in treating anxiety and panic disorders, obsessive compulsive disorders, and post-traumatic stress disorder [19]. While examining the effects of CBD, the cannabinoid was found to decrease autonomic arousal and conditioned fear expression, in addition to enhancing fear extinction and preventing long-term anxiogenic effects of stress [19]. However, the study specifically refers to acute instances and Blessing et al. [19],suggested more research be done in chronic CBD use in treatment. Additionally, Dimitriadis, Kapreli, Strimpakos, and Oldham [20]found anxiety to be closely associated with neck pain; which is indicative of the benefits of CBD in the treatment of pain, in addition to the prevention of anxiety caused by pain.

\section{Delta-9-tetrahydrocannabinol for the treatment of pain and other medical conditions}

Delta-9-tetrahydrocannabinol (THC) is recognized for its ability to reduce pain [17]. The literature has also suggested smoking cannabis is the most effective intake method that reduces neuropathic pain. 
Furthermore, nabiximol (specialized extract of cannabis) oromucosal sprays also reduced pain and improved sleep quality with varying clinical evidence $[1,21]$.

Additionally, Nabilone, a synthetic cannabinoid, is licensed in the United Kingdom and has been used for treatment of chemotherapy induced nausea and vomiting [22]. Individuals with neuropathic pain have also reported improvements in pain and sleep disturbance while using Nabilone[23]. Whereas dronabinol, another synthetic cannabinoid, has several analgesic effects on chronic pain with mixed results $[24,25]$. A phase I study by [24] showed that recipients of dronabinol experienced lessened pain intensity and increased satisfaction compared to the placebo group. In the phase II portion of the study participants were taking titrateddronabinol, in addition to their prescribed opioids. There was a significant increase in pain relief and satisfaction following the addition of dronabinol to their traditional opioid pain regimen [24].

Najicek et al. [25],investigated the effectiveness of cannabis extract in individuals with MS and determined that it was beneficial in the treatment of muscle stiffness [25]. The rate of relief was nearly twice as high when compared to the placebo group. After four and eight weeks the results were similar [25].

Comparatively, cannabinoids in the treatment of chronic pain have the potential to produce positive outcomes for therapeutic success with the decrease in neuropathic pain and spasticity. The ECS is described as an important facilitator of stress adaptation in the body. Changes in the ECS, such as up regulation of cannabinoid receptors increase probability that exogenous cannabinoids create a relaxing effect, as well as modulation of neuropathic pain and pain memory [26,27].

\section{Cannabidiol for the treatment of pain and other medical conditions}

Cannabidiol (CBD) has been recognized for its anti-inflammatory and antioxidant properties [28]. Cannabidiol lacks the psychotropic effects of THC, due to lack of intrinsic activity upon cannabinoid receptors [28]. Concerns with the use of psychoactive cannabinoids, such as THC, are risks of abuse and adverse side effects associated with higher doses [29]. For instance, a study by Ward et al. [30],revealed CBD did not result in adverse side effects or cognitive impairment following dosage of CBD for treatment of chronic induced neuropathic pain (CIPN), associated with Paclitaxel (PAC), in mice.

Moreover, it has been suggested that noninvasive drug delivery systems are needed to reduce pain symptoms [29]. Cannabidiol treatment provided the transdermal route is considered a non-addictive non-opioid therapy[29]. A study conducted by Paudel et al. [29], entailed the topical application of CBD to skin of hairless guinea pigs to determine its effectiveness when used via a transdermal delivery. The results demonstrated that there was a steady-state plasma concentration [29]. Results suggested that transdermal delivery of CBD is effective because of its high lipophilic property which allows the pharmacological agent to penetrate through the stratum corneum [29]. Paudel et al. [29], suggests that the use of CBD and alternate dosage forms can aide in reducing chronic pain, stating it could be a breakthrough treatment for individuals who do not respond well to available medications. A recent study introduced management of Multiple Sclerosis (MS) through application of topical CBD by investigating its therapeutic effects on autoimmune encephalomyelitis [31]. Results indicated that daily topical CBD (1\% CBD-cream) treatment may be neuroprotective against experimental autoimmune encephalomyelitis (EAE), a model of demyelinating diseases such as MS [31]. Researchers found that $\mathrm{CBD}$ was an effective treatment for regulating inflammatory pathways triggered by EAE by regulating pro-inflammatory cytokine secretions.

\section{Transdermal drug delivery}

Transdermal drug delivery is described as a topical non-invasive administration method of therapeutic agents[32]. Currently, enhanced transdermal delivery of substances into the body is not fully understood [11]. However, an understanding of the physiology of the skin enables researchers to identify the possible mechanism in which transdermal delivery of drugs is accomplished. The stratum corneum, the outermost skin layer, is composed of 10-15 layers of dead keratinocytes 10-20 micrometers thick, which are incorporated into a lipid bilayer matrix. This lipid matrix forms a permeation barrier that limits transdermal delivery only to small and moderately lipophilic molecules. The stratum corneum significantly influences the transdermal delivery of drugs. The functions of the stratum corneum include conserving water and electrolytes and selective permeability to certain substances [32]. Additionally, the epidermis and dermis layers of the skin contain proteolytic enzymes which act to degrade peptides and proteins [33].

Transdermal delivery enables controlled drug delivery through mechanisms, such as phonophoresis, which involves the use of ultrasound at a frequency of $20 \mathrm{kHZ}$ or greater to enhance delivery of molecules through the skin. In therapy the ultrasound frequencies used fortreatment range between 0.75 to $3.0 \mathrm{MHz} .3 \mathrm{MHz}$ is transmitted into the superficial tissues $(1-2 \mathrm{~cm})$ of the skin; $1 \mathrm{MHz}$ is transmitted into the deeper tissues $(3-5 \mathrm{~cm})$ of the skin and is an appropriate frequency for individuals with higher body fat [34]. The process involves the conversion of electrical energy into vibrational or sound energy, which is then used to permeabilize the skin [33]. Under a low frequency, phonophoresis is able to increase skin permeability to certain drugs and therapeutic compounds [32]. These compounds may be hydrophilic and/or have a large molecular weight. The mechanism by which phonophoresis increases skin permeability is not fully understood, however it has been suggested that cavitation is the primary mechanism involved because it formulates intercellular lipid channels and disorganization of lipid bilayers [32]. Cavitation may occur at either a high or low frequency [33]. The increased permeability is dependent on the development of an aqueous pathway across the stratum corneum, thus showing the importance of the penetration pathway for substances in transdermal delivery [32].

\section{Efficacy of phonophoresis for pain conditions}

Various studies have investigated the effectiveness of phonophoresis drug delivery on pain with mixed results. Shah and Dongre[12]conducted a study on the effectiveness of phonophoresis in De Quervain's disease. Phonophoresis when used with diclofenac (the medium), were reported to have a significant effect on pain and grip strength. The Gupta et al. [11], study reported that delivery of diclofenac sodium (the medium) via phonophoresis in a continuous ultrasonic mode was more effective in immediate pain relief than using a pulsated mode. Additionally, Yildiz et al. [35],reported that ketoprofen phonophoresis was effective in reducing pain when used 
alongside splinting for individuals with Carpal Tunnel Syndrome (CTS). However, the administration of solely ketoprofen phonophoresis did not prove to be better than the use of splinting alone for individuals with CTS. Additionally, the results were found to be inconclusive with previous research that had indicated both the ineffectiveness and effectiveness of phonophoresis [35]. A study conducted by Bakhtiary, Fatemi, Emami, and Malek[36], in which iontophoresis and phonophoresis methods were used to locally introduce dexamethasone sodium phosphate (Dex-P), an anti-inflammatory steroid drug, to a total of 34 individuals with CTS. Phonophoresis of Dex-P could elicit anti-inflammatory and tissue stimulating effects. Therapy was given for five minutes per session, five days a week for two weeks. Phonophoresis of Dex- $\mathrm{P}$ has the potential to accelerate normal resolution of inflammation and accelerate the healing process of damaged tissues. There was a significantly higher finger pinch and hand grip strength reported with the phonophoresis treatment. Dex-P phonophoresis resulted in a significant decrease in pain at the onset of treatment and four weeks post treatment. There was also an increase in the amplitude of motor and sensory action potential [36]. Durmus, Alayli, Tufekci, and Kuru[37]investigated and compared the efficacy of phonophoresis with capsaicin and therapeutic exercise, as well as placebo phonophoresis with capsaicin and therapeutic exercise, and therapeutic exercises on pain,disability, sleep quality, and depression of individuals with chronic neck pain. Each treatment showed significant improvements in each of the aforementioned areas. However, results regarding pain and disability were statistically significant in phonophoresis with capsaicin andplacebo phonophoresis with capsaicin when compared to therapeutic exercises alone. Phonophoresis used alongside therapeutic exercise may be used to clinically improve results as they relate to pain, disability, depression, and sleep quality [37]. In another study, Durmus et al. [38],investigated and compared the efficacy of phonophoresis and ultrasound therapy in the following areas for individuals with chronic low back pain: pain, disability, trunk muscle strength, walking performance, spinal mobility, quality of life, and depression. There were three groups which were given different treatments. The control groupwas given exercises alone. The second group received ultrasound and exercise and the third group received phonophoresis and exercises. Statistically significant improvements were reported for each group in the areas of pain, strength, endurance, 6-minute walking test, mobility, quality of life, and depression. The second and third group resulted in statistically significant differences of improvements in areas of pain, 6-minute walking test, and extensor muscle strength compared to the first group. Both ultrasound therapy and phonophoresis were effective in treating chronic low back pain; however, phonophoresis was more effective than ultrasound therapy [38]. Ay, Doğan, Evcik, and Baser [39]investigated the efficacy of phonophoresis, ultrasound therapy, and placebo ultrasound therapy in treating Myofascial Pain Syndrome (MPS). Individuals were divided into three treatment groups, in which all received neck exercises including isotonic, isometric, and stretching. The first group received phonophoresis with diclofenac. The second group received ultrasound and the third group received ultrasound therapy over trigger points. The following areas were assessed for each group: pain, neck Range of Motion (ROM), number of trigger points, algometric measurement, and disability. Results in phonophoresis and ultrasound groups indicated statistically significant improvements in pain severity, number of trigger points, pressure pain threshold, ROM, and neck pain disability index. Phonophoresis with diclofenac and ultrasound therapy proved to be effective in treating myofascial pain syndrome, however there were no significant differences in improvements between the first and second groups [39]. Soyupek et al. [40],investigated the efficacy of Phonophoresis with Non-steroid Anti-inflammatory Drugs (P-NSAID) and corticosteroid (P-CS) in CTS treatment, which was compared with the efficacy of phonophoresis with a Local Corticosteroid Injection (LCSI) and wrist splinting. The following areas were assessed: electrophysiological studies, hand dexterity, grip strength, sensory recovery of first three digits, and pain intensity. The administration of LCSI phonophoresis resulted in significant improvements in pain intensity and hand dexterity. However, there was a significant deterioration in the Semmes-Weinstein monofilament test and pegboard test. P-CS resulted in improvements in sensory recovery, distal latency, grip strength, and parameters of the DurozHand Index (DHI). P-NSAID resulted in significant improvement in grip strength, pegboard test, and pain intensity [40]. Lastly, the findings of Nagrale, Herd, Ganvir, and Rameke[41]demonstrate slight improvements regarding pain, pain-free grip, and functional status with the use of phonophoresis in clients with musculoskeletal conditions. Nagrale et al. [41],found that participants had improved outcomes from deep transverse friction massage, as opposed to phonophoresis

\section{Discussion}

There is therapeutic potential with the use of cannabinoids derived from the cannabis plant. THC has the ability to playa role in the reduction of chronic and neuropathic pain [1]. Studies have found that specialized extracts of cannabis, such as nabiximol and dronabinol, have been effective in pain relief $[21,24]$. CBD can reduce the effects of high glucose for individuals at risk for atherosclerosis, a risk factor for stroke [16] as well as treat anxiety for individuals with varying diagnoses such as post-traumatic diagnosis and panic disorders [19]. As it relates to this proposed therapeutic technique, the benefits of CBD, particularly when used topically, include the ability to reduce joint swelling due to its anti-inflammatory properties. CBD also has antioxidant properties andthese effects, along with the anti-inflammatory properties, can reduce pain [28].

Transdermal delivery of drugs has been implicated as an alternative pain reduction route due to its non-invasive properties [32]. Though the process of transdermal drug delivery is not fully understood, it is known that the stratum corneum plays an intricate role in this process [11]. Topicals such as CBD have been successful in transdermal delivery in mice and guinea pigs due the lipophilic properties of the cannabinoid which are known to penetrate through the selectively permeable stratum corneum [29,31]. Additionally, phonophoresis is classified as a transdermal drug delivery modality which involves the use of ultrasound to enhance transdermal delivery of molecules through the skin. The ultrasound frequencies used for treatment range between 0.75 to $3.0 \mathrm{MHz} .3 \mathrm{MHz}$ is used to treat superficial tissues, while $1 \mathrm{MHz}$ is used to target deeper tissues.

The mechanism referred to as cavitation, is a major factor during the transdermal drug delivery process because it increases the permeability of the skin through the development of cavities along with the expansion, contraction, and distortion of pre-existing gaseous bubbles within a liquid medium [42]. Mitragotri, Edwards, Blankschtein, and Langer[43] added that in order for phonophoresis to be successful in transporting agents transdermally it has to have the correct ultrasound frequency. Additional factors that enhance phonophoresis include: duty cycle, time of application, and intensity of ultrasound [44]. 
Therefore, phonophoresis has the potential to deliver drugs through a more controlled mechanism compared to topicals. Over the years, the use of phonophoresis as an agent to deliver medication in pain management has been studied. Studies have found that phonophoresis when used with various medications, alongside splinting, exercise, and ultrasound have alleviated pain symptoms, increased grip strength, and dexterity in individuals with De Quervain's disease, CTS, musculoskeletal and neurological conditions [11,12,35,37-40,].

Currently the literature regarding the efficacy of cannabinoids in pain management is quite limited. Similarly, the literature regarding the efficacy of phonophoresis in pain management is inconclusive. Furthermore, there is no literature regarding the use of phonophoresis as a modality to deliver cannabinoids as this is an innovative concept being introduced in this scoping review. Therefore, further research is warranted to determine patient outcomes in regard to the reduction of pain, with the use of phonophoresis as a modality to deliver cannabinoids.

\section{Recommendation: Delivery of Cannabinoids Through Phonophoresis}

Cannabidiol (CBD) and THC oils are non-water soluble and classified as hydrophobic substances [45]. Therefore, because these oils are hydrophobic, delivery into the stratum corneum's selectively permeable lipid bilayer is problematic. However, due to advancements in the medical cannabis industry there has been development of water compatiblecannabinoids [45]. To elaborate, CBD and THC are capable of becoming water compatible through nanoemulsions, which are stable homogeneous mixtures of water and oil. Nano-cannabinoids can be rapidly absorbed into the body either orally or into the skin. Rapid absorption increases potency and onset action, which enhances the therapeutic effect.Nanoemulsions are made with a lower amount of surfactants, which significantly dilutes the mixture [45]. Nanoemulsions are formulated with significantly lower amounts of surfactants compared to microemulsions and liposomes [46].

Properties of nanoemulsions include low viscosity, high interfacial surface area and long-term kinetic stability, as well as being completely water-soluble [46] Nano-emulsifications may be created by ultrasound, which utilizes cavitation to break up cannabis extract droplets down to a nanometer [47]. The CBD/THC nanoemulsion mixture formula consists of cannabis oil extract $(5.40 \%)$, carrier oil (7.20\%), surfactant $(2.00 \%)$, and water $(85.4 \%)$ [ 47$]$. Therefore, this innovative nanoemulsion has the potential to be effective in transdermal drug delivery through phonophoresis.

\section{Implications for the Health Care Profession and Society}

Utilizing cannabis and cannabinoids to treat medical illnesses has become more prevalent in the United States and globally [48]. Society is becoming interested in the use of cannabinoids to reduce pain. These herbal agents are facilitating the understanding of pain mechanisms (Orthopedics, 2008). Several states in the United States have passed laws approving the use of cannabis for medicinal purposes, resulting in decriminalization of its use [49]. Likewise, physicians are now permitted to discuss cannabis treatments with patients, [50]. The use of cannabinoids may provide improvement in patient care, primarily as supportive agents orprimary interventions [50]. Conversely, while cannabis may have many positive therapeutic effects on individuals, it has been reported that smoking marijuana regularly can increase one's risk of lung and cardiovascular problems, due to the general effects of smoking [17]. In order to prevent lung and cardiovascular conditions, other methods of ingestion are recommended, such as oral, sublingual, and topical methods. phonophoresisInaddition, research has indicated that long-term use of medicinal cannabinoids lack dependency, tolerance and mental health side effects. Individuals should be aware that new central nervous system symptoms may not be caused by the cannabinoid therapy, but from underlying diseases or complications [51].

The biomechanical model focuses on accurately measuring and addressing limitations of functional movements that cause problems to the musculoskeletal capacities and functional motion in everyday occupational performances. This model can be used by occupational therapists as a reference to develop therapeutic treatments, reduce pain, and facilitate engagement in occupational performance [52] The biomechanical model in conjunction with phonophoresis and medicinal cannabinoids ointments can be used to reduce pain; prevent deformities by maintaining existing capacities of motion; restore motion capacities, and compensating for limited motion, strength and endurance[52]. In today's healthcare culture, the use of medicinal cannabinoids is relatively new. As new therapeutic areas emerge in occupational therapy and the confidence in its administration increases there is still more to learn regarding the therapeutic potential of cannabinoids.

\section{Limitations}

The proposed therapeutic technique may be hampered by several limitations which involve issues of legality and societal acceptance of cannabinoid use. Cannabinoids are not federally legal and are in fact classified as a Schedule I drug-controlled substance, which are subject to federal research limitations [53]. The American Society of Health-System Pharmacists and the American Medical Association have recommended that there be a modification of the Schedule I status of marijuana in order for research to be conducted properly, thus permitting for the development of evidence-based societal and public health policies, as well as clinical management strategies [54]. Lastly, anti-legalization arguments emphasize potential public health consequences such as: the potential harm on youth health, marijuana-impaired driving, and the development of an industry that puts profit before public health, such as the tobacco industry [55].

\section{Conclusion}

Cannabis has been used for medicinal purposes for over 5,000 years. The plant has been used throughout the years to treat multiple conditions such as rheumatic pain, anxiety, spasticity, multiple sclerosis, complex regional pain syndrome, as well as numerous other conditions. Phonophoresis has been utilized to deliver pharmacological agents such as Dex-P, non-steroid anti-inflammatory drugs (N-SAIDs), capsaicin, corticosteroid, local corticosteroid injections in the treatment of pain-related conditions. Although, both cannabis and phonophoresis have therapeutic potential, they have yet to be used in conjunction with one another. Therefore, this scoping review was conducted in order to review the current literature on cannabinoids and phonophoresis, identify gaps in the literature, and introduce the therapeutic potential of transdermal delivery of cannabinoids through phonophoresis. The transdermal delivery of pharmacological cannabinoids through phonophoresis may be possible through 
Citation: Hunnicutt C, Grullón E, Morrison M, Lomeli I, Hubbard K (2019) The Therapeutic Potential of Cannabinoids and Phonophoresis in Pain Management: A Scoping Review. J Phys Med Rehabil Disabil 5: 036.

nano-emulsifications of THC and CBD. Furthermore, the process of phonophoresis allows the cannabinoids to be delivered to specific peripheral sites. The therapeutic use of cannabinoids is a relatively new area and further research is recommended. Additionally, research on phonophoresis has demonstrated inconclusive results associated with pain management. Efficacy is dependent on the size and chemical properties of the anti-inflammatory or analgesic agent being delivered. Thus, further research is recommended regarding the efficacy of phonophoresis for transdermal delivery of certain drugs.

\section{References}

1. Jensen B, Chen J, Furnish T, Wallace M (2015) Medical marijuana and chronic pain: A review of basic science and clinical evidence. Curr Pain Headache Rep 19: 524.

2. Khaiser M, Meiko P, Ahrari S, Pasetka M, DeAngelis C (2016) Medical cannabis dosing strategies in pain-related conditions: A scoping review of current literature. Journal of Pain Management 9: 449-463.

3. National Conference of State Legislatures (2019) State medical marijuana laws. Washington, D.C,USA.

4. Hutton H (2014) Beyond THC: Exploring the topical uses of cannabis. Journal of the American Herbalists Guild 12: 40-44.

5. Temple LM (2016) Medical marijuana and pain management. Diseasea-Month 62: 346-352.

6. Battista N, Di Tommaso M, Bari M, Maccarrone M (2012) The endocannabinoid system: An overview. Frontiers in Behavioral Neuroscience Journal, 6: 1-7.

7. Abramovici H (2013) Information for health care professionals: Cannabis (marihuana, marijuana) and the cannabinoids: Dried or fresh plant and oil for administration by ingestion or other means psychoactive agent.

8. Borgelt LM, Franson KL, Nussbaum AM, Wang GS (2013) The pharmacological and clinical effects of medical cannabis. Pharmacotherapy 33: $195-209$

9. Rao R, Nanda S (2009) Sonophoresis: Recent advancements and future trends. J Pharm Pharmacol 61: 689-705.

10. Abrahams S (2008) Phonophoresis of non-steroidal drugs: A review of clinical evidence. International Musculoskeletal Medicines 30: 37-41.

11. Gupta C, Rajput M, Samuel A, Kalra S (2013) Phonophoresis in continuous mode ultrasound has significant effect in the relieving pain in upper trapezius tender point. Indian Journal of Physiotherapy \& Occupational Therapy 7: 26-30.

12. Shah H, Dongre B (2014) A Study to Determine the effect of Ultrasound and Phonophoresis in De Quervain's Diseases. Indian Journal of Physiotherapy \& Occupational Therapy 8: 224-229.

13. Arksey H, O’Malley L (2005) Scoping studies: Towards a methodological framework. International Journal of Social Research Methodology 8: 19-32.

14. Syed YY, McKeage K, Scott LJ (2014) Delta-9-Tetrahdrocannabinol/ cannabidiol (Sativex): A review of its use in patients with moderate to severe spasticity due to multiple sclerosis. Drugs $74: 563-578$.

15. Drossel C, Forchheimer M, Meade MA (2016) Characteristics of individuals with spinal cord injury who use cannabis for therapeutic purposes. Topics in Spinal Cord Injury Rehabilitation 22: 3-12.

16. Rajesh M, Mukhopadhyay P, Bátkai S, Haskó G, Liaudet L, et al. (2007) Cannabidiol attenuates high glucose-induced endothelial cell inflammatory response and barrier disruption. Am J Physiol Heart CircPhysiol 293: 610-619.
17. Macdougall AK (2017) Cannabis for Arthritis? Medical marijuana may bring some relief - but evidence is sketchy. Arthritis Today 31: 39 .

18. Hammell D, Zhang L, Ma F, Abshire S, McIlwrath S, et al. (2016) Transdermal cannabidiol reduces inflammation and pain-related behaviours in a rat model of arthritis. Eur J Pain 20: 936-948.

19. Blessing EM, Steenkamp MM, Manzanares J, Marmar CR (2015) Cannabidiol as a potential treatment for anxiety disorders. Neurotherapeutics 12: 825-836.

20. Dimitriadis Z, Kapreli E, Strimpakos N, Oldham J (2015) Do psychological states associate with pain and disability in chronic neck pain patients? Journal of Back and Musculoskeletal Rehabilitation 28: 797-802.

21. Hwang JK, Clarke H (2016) Cannabis and pain: A review. Journal of Pain Management 9: 395-413.

22. Frank B, Serpell MG, Hughes J, Matthews JN, Kapur D (2008) Comparison of analgesic effects and patient tolerability of nabilone and dihydrocodeine for chronic neuropathic pain: Randomized, crossover, double blind study. BMJ 336: 199-201.

23. Bestard JA, Toth CC (2011) An open-label comparison of nabilone and gabapentin as adjuvant therapy or monotherapy in the management of neuropathic pain in patients with peripheral neuropathy. Pain Practice, 11: 353-368.

24. Narang S, Gibson D, Wasan AD, Ross EL, Michna E, et al. (2007) Efficacy of dronabinol as an adjuvant treatment for chronic pain patients on opioid therapy. J Pain 9: 254-264.

25. Zajicek JP, Hobart JC, Slade A, Barnes D, Mattison PG (2012) Multiple sclerosis and extract of cannabis: results of the MUSEC trial. J NeurolNeurosurg Psychiatry 83: 1125-1132.

26. KarPaudel KS, Hammell DC, Agu RU, Valiveti S, Stinchcomb AL (2010) Cannabidiol bioavailability after nasal and transdermal application: effect of permeation enhancers. Drug Dev Ind Pharm 36: 1088-1097.

27. st M, Wippermann S, Ahrens J (2010) Role of cannabinoids and the treatment of pain and (painful) spasticity. Drugs 70: 2409-2438.

28. Kaufmann RM, Kraft B, Frey R, Winkler D, Weiszenbichler S, et al. (2009) Acute psychotropic effects of oral cannabis extract with a defined content of Delta9-tetrahydrocannabinol (THC) in healthy volunteers. Pharmacopsychiatry 43: 24-32.

29. Scuderi C, Filippis DD, Iuvone T, Blasio A, Steardo A, et al. (2009) Cannabidiol in medicine: a review of its therapeutic potential in CNS disorders. Phytother Res 23: 597-602.

30. Ward SJ, McAllister SD, Kawamura R, Murase R, Neelakantan H, et al. (2014) Cannabidiol inhibits paclitaxel-induced neuropathic pain through 5-HT(1A) receptors without diminishing nervous system function or chemotherapy efficacy. Br J Pharmacol 171: 636-645.

31. Giacoppo S, Galuppo M, Pollastro F, Grassi G, Bramanti P, et al. (2015) A new formulation of cannabidiol in cream shows therapeutic effects in a mouse model of experimental autoimmune encephalomyelitis. Daru 23: 48 .

32. Park D, Park H, Seo J, Lee S (2014) Sonophoresis in transdermal drug delivery's. Ultrasonics 54: 56-65.

33. Singh N, Kalluri H, Herwadkar A, Badkar A, Banga AK (2012) Transcending the skin barrier to deliver peptides and proteins using active technologies. Crit Rev Ther Drug Carrier Syst 29: 265-298.

34. Allen LV Compounding for phonophoresis. SecundumArtem: Current \& Compounding Information for the Pharmacist.

35. Yildiz N, Atalay NS, Gungen GO, Sanal E, Akkaya N, et al. (2011) Comparison of ultrasound and ketoprofen phonophoresis in the treatment of carpal tunnel syndrome. J Back Musculoskelet Rehabil 24: 39-47. 
36. Bakhtiary HA, Fatemi E, Emami M, Malek M (2013) Phonophoresis of Dexamethasone sodium phosphate may manage pain and symptoms of patients with carpal tunnel syndrome (CTS). Clinical Journal of Pain 29: 348-53.

37. Durmus D, Alayli G, Tufekci T, Kuru O (2014) A randomized placebo-controlled clinical trial of phonophoresis for the treatment of chronic neck pain. Rheumatology International 34: 605-611.

38. Durmus D, Alayli G, Goktepe AS, Taskaynatan MA, Bilgici A et al., (2013) Is phonophoresis effective in the treatment of chronic low back pain: A single-blind randomized controlled trial. Rheumatology International 33: 1737-1744.

39. Ay S, Doğan SK, Evcik D, Başer OC (2011) Comparison the efficacy of phonophoresis and ultrasound therapy in myofascial pain syndrome. Rheumatology International 3: 1203-1208.

40. Soyupek F, Kutluhan S, Uslusoy G, Ilgun E, Eris S, et al. (2012) The efficacy of phonophoresis on electrophysiological studies of the patients with carpal tunnel syndrome. Rheumatol Int 32: 3235-3242.

41. Nagrale AV, Herd CR, Ganvir S, Ramteke G (2009) Cyriax physiotherapy versus phonophoresis with supervised exercise in subjects with lateral epicondylalgia: A randomized clinical trial. J Man ManipTher 17: 171-178.

42. Leighton TG (1997) The principles of cavitation. Chapter 9 of Povey MJW, Mason TJ(eds.). Ultrasound in food processing, Thomson Science, London, Blackie Academic and Professional Pg no: 151-182.

43. Mitragotri S, Edwards DA, Blankschtein D, Langer R (1995) A mechanistic study of ultrasonically-enhanced transdermal drug delivery. J Pharm Sci 84: 697-706.

44. Tezel A, Sens A, Tuchscherer J, Mitragotri S (2001) Frequency dependence of sonophoresis. Pharm Res 18: 1694-1700.

45. Peshkovsky A (2016b) Can CBD or THC be made water-soluble? Sonomechanics blog.
46. PeshkovskyA (2016a) Are cannabinoids more effective in nano form? Sonomechanics blog.

47. PeshkovskyA(2016c) Water-soluble cannabis oils: Microemulsion, liposomes or nanoemulsion? Sonomechanics blog.

48. Hill KP (2015) Medical marijuana for treatment of chronic pain and other medical and psychiatric problems: A clinical review. JAMA 313: 2474-2483.

49. Goldenberg M, Reid MW, Ishak WW, Danovitch I (2017) The impact of cannabis and cannabinoids for medical conditions on health-related quality of life: A systematic review and meta-analysis. Drug Alcohol Depend 174: 80-90.

50. McCarberg BH (2007) The treatment of breakthrough pain. Pain Medicine 8: 8-13.

51. Notcutt WG (2015) Clinical use of cannabinoids for symptom control in multiple sclerosis. Neurotherapeutics (12): 769-777.

52. Kielhofner G (2009) Conceptual foundations of occupational therapy practice. Philadelphia PA: F.A. Davis Company.

53. McGinty EE, Niederdeppe J, Heley K, Barry CL (2017) Public perceptions of arguments supporting and opposing recreational marijuana legalization. Prev Med 99: 80-86.

54. Krenzelok EP (2017) Marijuana legalization: A continuing conundrum. Am J Health Syst Pharm 74: 1843-1844.

55. McGinty EE, Samples H, Bandara SN, Saloner B, Bachhuber MA, et al. (2016) The emerging public discourse on state legalization of marijuana for recreational use in the US: Analysis of news media coverage, 20102014. Prev Med 90: 114-120. 


\section{Hit}

Journal of Anesthesia \& Clinical Care

Journal of Addiction \& Addictive Disorders

Advances in Microbiology Research

Advances in Industrial Biotechnology

Journal of Agronomy \& Agricultural Science

Journal of AIDS Clinical Research \& STDs

Journal of Alcoholism, Drug Abuse \& Substance Dependence

Journal of Allergy Disorders \& Therapy

Journal of Alternative, Complementary \& Integrative Medicine

Journal of Alzheimer's \& Neurodegenerative Diseases

Journal of Angiology \& Vascular Surgery

Journal of Animal Research \& Veterinary Science

Archives of Zoological Studies

Archives of Urology

Journal of Atmospheric \& Earth-Sciences

Journal of Aquaculture \& Fisheries

Journal of Biotech Research \& Biochemistry

Journal of Brain \& Neuroscience Research

Journal of Cancer Biology \& Treatment

Journal of Cardiology: Study \& Research

Journal of Cell Biology \& Cell Metabolism

Journal of Clinical Dermatology \& Therapy

Journal of Clinical Immunology \& Immunotherapy

Journal of Clinical Studies \& Medical Case Reports

Journal of Community Medicine \& Public Health Care

Current Trends: Medical \& Biological Engineering

Journal of Cytology \& Tissue Biology

Journal of Dentistry: Oral Health \& Cosmesis

Journal of Diabetes \& Metabolic Disorders

Journal of Dairy Research \& Technology

Journal of Emergency Medicine Trauma \& Surgical Care

Journal of Environmental Science: Current Research

Journal of Food Science \& Nutrition

Journal of Forensic, Legal \& Investigative Sciences

Journal of Gastroenterology \& Hepatology Research

Journal of Gerontology \& Geriatric Medicine
Journal of Genetics \& Genomic Sciences

Journal of Hematology, Blood Transfusion \& Disorders

Journal of Human Endocrinology

Journal of Hospice \& Palliative Medical Care

Journal of Internal Medicine \& Primary Healthcare

Journal of Infectious \& Non Infectious Diseases

Journal of Light \& Laser: Current Trends

Journal of Modern Chemical Sciences

Journal of Medicine: Study \& Research

Journal of Nanotechnology: Nanomedicine \& Nanobiotechnology

Journal of Neonatology \& Clinical Pediatrics

Journal of Nephrology \& Renal Therapy

Journal of Non Invasive Vascular Investigation

Journal of Nuclear Medicine, Radiology \& Radiation Therapy

Journal of Obesity \& Weight Loss

Journal of Orthopedic Research \& Physiotherapy

Journal of Otolaryngology, Head \& Neck Surgery

Journal of Protein Research \& Bioinformatics

Journal of Pathology Clinical \& Medical Research

Journal of Pharmacology, Pharmaceutics \& Pharmacovigilance

Journal of Physical Medicine, Rehabilitation \& Disabilities

Journal of Plant Science: Current Research

Journal of Psychiatry, Depression \& Anxiety

Journal of Pulmonary Medicine \& Respiratory Research

Journal of Practical \& Professional Nursing

Journal of Reproductive Medicine, Gynaecology \& Obstetrics

Journal of Stem Cells Research, Development \& Therapy

Journal of Surgery: Current Trends \& Innovations

Journal of Toxicology: Current Research

Journal of Translational Science and Research

Trends in Anatomy \& Physiology

Journal of Vaccines Research \& Vaccination

Journal of Virology \& Antivirals

Archives of Surgery and Surgical Education

Sports Medicine and Injury Care Journal

International Journal of Case Reports and Therapeutic Studies

Journal of Ecology Research and Conservation Biology

Submit Your Manuscript: http://www.heraldopenaccess.us/Online-Submission.php 